\title{
Some properties of Fibonacci and Chebyshev polynomials
}

Yang $\mathrm{Li}^{*}$

\section{*Correspondence:}

ly13119143216@126.com Department of Mathematics,

Northwest University, Xi'an, Shaanxi, P.R. China

\begin{abstract}
In this paper, we study the properties of Chebyshev polynomials of the first and second kind and those of Fibonacci polynomials and use an elementary method to give Chebyshev polynomials of the first and second kind in terms of Fibonacci polynomials and vice versa. Finally, we get some identities involving the Fibonacci numbers and the Lucas numbers.
\end{abstract}

Keywords: elementary method; Chebyshev polynomials; Fibonacci polynomials; Fibonacci numbers; Lucas numbers

\section{Introduction}

As we know, the Chebyshev polynomials and Fibonacci polynomials are usually defined as follows: Chebyshev polynomials of the first kind are $T_{n+2}(x)=2 x T_{n+1}(x)-T_{n}(x), n \geq 0$, with the initial values $T_{0}(x)=1, T_{1}(x)=x$; Chebyshev polynomials of the second kind are $U_{n+2}(x)=2 x U_{n+1}(x)-U_{n}(x), n \geq 0$, with the initial values $U_{0}(x)=1, U_{1}(x)=2 x$; Fibonacci polynomials are $F_{n+2}(x)=x F_{n+1}(x)+F_{n}(x), n \geq 0$, with the initial values $F_{0}(x)=0, F_{1}(x)=1$. From the second-order linear recurrence sequences, we have

$$
\begin{aligned}
& T_{n}(x)=\frac{1}{2}\left[\left(x+\sqrt{x^{2}-1}\right)^{n}+\left(x-\sqrt{x^{2}-1}\right)^{n}\right], \\
& U_{n}(x)=\frac{1}{2 \sqrt{x^{2}-1}}\left[\left(x+\sqrt{x^{2}-1}\right)^{n+1}-\left(x-\sqrt{x^{2}-1}\right)^{n+1}\right], \\
& F_{n}(x)=\frac{1}{2^{n} \sqrt{x^{2}+4}}\left[\left(x+\sqrt{x^{2}+4}\right)^{n}-\left(x-\sqrt{x^{2}+4}\right)^{n}\right] .
\end{aligned}
$$

These polynomials play a very important role in the study of the theory and application of mathematics, and they are closely related to the famous Fibonacci numbers $\left\{F_{n}\right\}$ and Lucas numbers $\left\{L_{n}\right\}$ which are defined by the second-order linear recurrence sequences

$$
\begin{aligned}
& F_{n+2}=F_{n+1}+F_{n}, \\
& L_{n+2}=L_{n+1}+L_{n},
\end{aligned}
$$

where $n \geq 0, F_{0}=0, F_{1}=1, L_{0}=2$ and $L_{1}=1$. Therefore, many authors have investigated these polynomials and got many properties and corollaries. For example, Zhang [1] uses

\section{照 Springer}

(c) $2015 \mathrm{Li}$ : licensee Springer. This is an Open Access article distributed under the terms of the Creative Commons Attribution License (http://creativecommons.org/licenses/by/4.0), which permits unrestricted use, distribution, and reproduction in any medium, provided the original work is properly credited. 
the Chebyshev polynomials and has obtained the general formulas involving $F_{n}$ and $L_{n}$,

$$
\begin{aligned}
& \sum_{a_{1}+a_{2}+\cdots+a_{k+1}=n} F_{m\left(a_{1}+1\right)} \cdot F_{m\left(a_{2}+1\right)} \cdots F_{m\left(a_{k+1}+1\right)} \\
= & (-i)^{m n} \frac{F_{m}^{k+1}}{2^{k} \cdot k !} U_{n+k}^{(k)}\left(\frac{i^{m} L_{m}}{2}\right), \\
& \sum_{a_{1}+a_{2}+\cdots+a_{k+1}=n+k+1} L_{m\left(a_{1}+1\right)} \cdot L_{m\left(a_{2}+1\right)} \cdots L_{m\left(a_{k+1}+1\right)} \\
= & (-i)^{m(n+k+1)} \frac{2}{k !} \sum_{h=0}^{k+1}\left(\frac{i^{m+2} L_{m}}{2}\right)^{h} \frac{(k+1) !}{h !(k+1-h) !} U_{n+2 k+1-h}^{(k)}\left(\frac{i^{m} L_{m}}{2}\right),
\end{aligned}
$$

where $k, m$ are any positive integers, $a_{1}, a_{2}, \ldots, a_{k+1}$ are nonnegative integers and $i$ is the square root of -1 . Falcón and Plaza [2,3] presented many formulas about Fibonacci polynomials. This fact allowed them to present a family of integer sequences in a new and direct way. Zhang [1] also used the Chebyshev polynomials to solve some calculating problems of the general summations. Wu and Yang [4] studied Chebyshev polynomials and got a lot of properties.

In this paper, we combine Sergio Falcón and Wenpeng Zhang's ideas. Then we obtain the following theorems and corollaries. These results strengthen the connections of two kinds of polynomials. They are also helpful in dealing with some calculating problems of the general summations or studying some integer sequences.

Theorem 1 For any positive integer $n$, we have the identities

$$
\begin{aligned}
& T_{2 n}(x)=\sum_{s=1}^{n+1} \sum_{k=0}^{n} \frac{2^{2 k} \cdot(2 s n-n)(n+k-1) ! F_{2 s-1}(x)}{(-1)^{s+n-1}(n-k) !(k+s) !(k-s+1) !} \\
& T_{2 n-1}(x)=\sum_{s=1}^{n} \sum_{k=1}^{n} \frac{2^{2 k-1}(2 n s-s)(n+k-2) ! F_{2 s}(x)}{(-1)^{s+n}(n-k) !(k+s) !(k-s) !} .
\end{aligned}
$$

Theorem 2 For any positive integer $n$, we have

$$
\begin{aligned}
& U_{2 n}(x)=\sum_{s=1}^{n+1} \sum_{k=0}^{n} \frac{2^{2 k-1}(1-2 s)(n+k) ! F_{2 s-1}(x)}{(-1)^{s+n}(n-k) !(k+s) !(k-s+1) !}, \\
& U_{2 n-1}(x)=\sum_{s=1}^{n} \sum_{k=0}^{n-1} \frac{2^{2 k+2} s(n+k-1) !(-1)^{s+n} F_{2 s}(x)}{(n-k+1) !(k+s+1) !(k-s+1) !}
\end{aligned}
$$

Theorem 3 For any positive integer $n$, we have the following forms:

$$
\begin{aligned}
& F_{2 n-1}(x)=\sum_{k=0}^{n-1} \sum_{j=1}^{n-1} \frac{2^{2 j+2-2 n}(2 k+1)(n-j-1) !}{j !(n-k-j-1) !(n+k-j) !} U_{2 k}(x), \\
& F_{2 n}(x)=\sum_{k=1}^{n-1} \sum_{j=1}^{n-1} \frac{2^{2 j-2 n+2} k(2 n-j-1) !}{j !(n-k-j) !(n+k-j) !} U_{2 k}(x) .
\end{aligned}
$$


Theorem 4 For any positive integer $n$, we have the following forms:

$$
\begin{aligned}
& F_{2 n}(x)=\sum_{k=1}^{n} \sum_{j=1}^{n-1} \frac{2^{2 j+2-2 n}(2 n-j-1) ! T_{2 k-1}(x)}{j !(2 n+2 k-2 j-2) !(2 n-2 k-2 j) !}, \\
& F_{2 n-1}(x)=\sum_{k=1}^{n-1} \sum_{j=1}^{n-1} \frac{2^{2 j+3-2 n}(2 n-j-2) ! T_{2 k}(x)}{j !(n+k-j-1) !(n-k-j-1) !}+\sum_{j=1}^{n-1} \frac{2^{2 j+2-2 n}(2 n-j-2) !}{j !(n-j-1) !(n-j-1) !} .
\end{aligned}
$$

Corollary 1 For any positive integer $n$, we have the following identities:

$$
\begin{aligned}
& F_{2 n-1}\left(\frac{i^{m} L_{m}}{2}\right)=\sum_{k=0}^{n-1} \sum_{j=1}^{n-1} \frac{2^{2 j+2-2 n}(2 k+1)(n-j-1) ! F_{m(2 k+1)}}{j !(n-k-j-1) !(n+k-j) !(-1)^{k m} F_{m}}, \\
& F_{2 n}\left(\frac{i^{m} L_{m}}{2}\right)=\sum_{k=1}^{n-1} \sum_{j=1}^{n-1} \frac{2^{2 j-2 n+2} k(2 n-j-1) !(-i) F_{2 k m}}{(-1)^{k m} j !(n-k-j) !(n+k-j) ! F_{m}} .
\end{aligned}
$$

Corollary 2 For any positive integer $n$, we have the following identities:

$$
\begin{aligned}
F_{2 n}\left(\frac{i^{m} L_{m}}{2}\right)= & \sum_{k=1}^{n} \sum_{j=1}^{n-1} \frac{2^{2 j+2-2 n}(2 n-j-1) !(-1)^{k m}(-i) L_{2 k m-m}}{j !(2 n+2 k-2 j-2) !(2 n-2 k-2 j) !}, \\
F_{2 n-1}\left(\frac{i^{m} L_{m}}{2}\right)= & \sum_{k=1}^{n-1} \sum_{j=1}^{n-1} \frac{2^{2 j+3-2 n}(2 n-j-2) !(-1)^{k m} L_{2 k m}}{j !(n+k-j-1) !(n-k-j-1) !} \\
& +\sum_{j=1}^{n-1} \frac{2^{2 j+2-2 n}(2 n-j-2) !}{j !(n-j-1) !(n-j-1) !}
\end{aligned}
$$

\section{Some lemmas}

Lemma 1 For any nonnegative integers $m$ and $n$, we have these identities

$$
\begin{aligned}
& \int_{-1}^{1} \frac{T_{m}(x) T_{n}(x)}{\sqrt{1-x^{2}}} d x= \begin{cases}0, & m \neq n, \\
\frac{\pi}{2}, & m=n>0, \\
\pi, & m=n=0 ;\end{cases} \\
& \int_{-1}^{1} U_{m}(x) U_{n}(x) \sqrt{1-x^{2}} d x= \begin{cases}0, & m \neq n, \\
\frac{\pi}{2}, & m=n ;\end{cases} \\
& T_{n}(\cos \theta)=\cos n \theta ; \\
& U_{n}(\cos \theta)=\frac{\sin (n+1) \theta}{\sin \theta} .
\end{aligned}
$$

Proof See reference [5].

Lemma 2 For any positive integers $m$ and $n$, we have these identities

$$
\begin{aligned}
& U_{n}\left(\frac{i}{2}\right)=i^{n} F_{n+1}, \\
& T_{n}\left(\frac{i}{2}\right)=\frac{i^{n}}{2} L_{n+1},
\end{aligned}
$$




$$
\begin{aligned}
& T_{n}\left(T_{m}\left(\frac{i}{2}\right)\right)=i^{m n} L_{m n}, \\
& U_{n}\left(T_{m}\left(\frac{i}{2}\right)\right)=i^{m n} \frac{F_{m(n+1)}}{F_{m}} .
\end{aligned}
$$

Proof See reference [1].

Lemma 3 For any positive integer $n$, we have

$$
\begin{aligned}
& T_{n}\left(T_{m}(x)\right)=T_{n m}(x), \\
& U_{n}\left(T_{m}(x)\right)=\frac{U_{m(n+1)-1}(x)}{U_{m-1}(x)} .
\end{aligned}
$$

Proof See reference [1].

Lemma 4 For any positive integer $n$, let

$$
F_{n}(x)=\sum_{k=0}^{+\infty} a_{n k} U_{k}(x)
$$

and

$$
F_{n}(x)=\frac{1}{2} b_{n 0} T_{0}(x)+\sum_{k=1}^{+\infty} b_{n k} T_{k}(x)
$$

then we can get

$$
\begin{aligned}
& a_{n k}= \begin{cases}\sum_{j=1}^{\left\lfloor\frac{n-1}{2}\right\rfloor} \frac{2(k+1)(n-j-1) !}{j !(n-k-2 j-1) !(n+k+1-2 j) ! !}, & k+n \text { is odd }, \\
0, & \text { otherwise, }\end{cases} \\
& b_{n k}= \begin{cases}\sum_{j=1}^{\left\lfloor\frac{n-1}{2}\right\rfloor} \frac{2(n-j-1) !}{j !(n+k-2 j-1) !(n-k-2 j-1) ! !}, & k+n \text { is odd, } \\
0, & \text { otherwise. }\end{cases}
\end{aligned}
$$

Proof To begin with, we multiply $\sqrt{1-x^{2}} U_{m}(x)$ to both sides of (5), then integrate it from -1 to 1 , we can get the following identity by applying property (2):

$$
\int_{-1}^{1} \sqrt{1-x^{2}} F_{n}(x) U_{m}(x) d x=\sum_{k=1}^{\infty} \int_{-1}^{1} a_{n k} \sqrt{1-x^{2}} U_{m}(x) U_{k}(x) d x=\frac{\pi}{2} a_{n m},
$$

and then we have

$$
a_{n m}=\frac{2}{\pi} \int_{-1}^{1} \sqrt{1-x^{2}} F_{n}(x) U_{m}(x) d x
$$

From reference [2] we know

$$
F_{n}(x)=\sum_{j=0}^{\left\lfloor\frac{n-1}{2}\right\rfloor}\left(\begin{array}{c}
n-j-1 \\
j
\end{array}\right) x^{n-2 j-1}
$$


where $n \geq 1$. We define

$$
w_{n k}=\frac{2}{\pi} \int_{0}^{\pi} \cos ^{n} \theta \sin (k+1) \theta \sin \theta d \theta .
$$

From reference [6] we know

$$
w_{n k}= \begin{cases}\frac{2(k+1) n !}{(n+k+2) !(n-k) ! !}, & k+n \text { is even and } n \geq k \\ 0, & \text { otherwise }\end{cases}
$$

where $n$ and $k$ are any nonnegative integers. Let $x=\cos \theta$, then we can get the following identity by applying property (2) and property (9):

$$
\begin{aligned}
a_{n m} & =\frac{2}{\pi} \int_{0}^{\pi} F_{n}(\cos \theta) \sin (m+1) \theta \sin \theta d \theta \\
& =\frac{2}{\pi} \sum_{j=1}^{\left\lfloor\frac{n-1}{2}\right\rfloor}\left(\begin{array}{c}
n-j-1 \\
j
\end{array}\right) \int_{0}^{\pi} \cos ^{n-2 j-1} \theta \sin (m+1) \theta \sin \theta d \theta \\
& =\sum_{j=1}^{\left\lfloor\frac{n-1}{2}\right\rfloor}\left(\begin{array}{c}
n-j-1 \\
j
\end{array}\right) w_{n-2 j-1, m},
\end{aligned}
$$

and then we have $a_{n m}=0$ if $n+m$ is even. If $n+m$ is odd, we have

$$
\begin{aligned}
a_{n m} & =\sum_{j=1}^{\left\lfloor\frac{n-1}{2}\right\rfloor}\left(\begin{array}{c}
n-j-1 \\
j
\end{array}\right) \cdot \frac{2(m+1)(n-2 j-1) !}{(n-m-2 j-1) ! !(n+m+1-2 j) ! !} \\
& =\sum_{j=1}^{\left\lfloor\frac{n-1}{2}\right\rfloor} \frac{(n-j-1) !}{j !(n-2 j-1) !} \cdot \frac{2(m+1)(n-2 j-1) !}{(n-m-2 j-1) ! !(n+m+1-2 j) ! !} \\
& =\sum_{j=1}^{\left\lfloor\frac{n-1}{2}\right\rfloor} \frac{2(m+1)(n-j-1) !}{j !(n-m-2 j-1) ! !(n+m+1-2 j) ! !} .
\end{aligned}
$$

We finish proving property (7).

In order to prove property (8), we must multiply $\frac{T_{m}(x)}{\sqrt{1-x^{2}}}$ to both sides of (6), then integrate it from -1 to 1 , we can get the following identity by applying property (1):

$$
\int_{-1}^{1} \frac{F_{n}(x) T_{m}(x)}{\sqrt{1-x^{2}}} d x=\int_{-1}^{1} \frac{b_{n 0} T_{0}(x) T_{m}(x)}{2 \sqrt{1-x^{2}}} d x+\sum_{k=1}^{\infty} \int_{-1}^{1} \frac{b_{n k} T_{m}(x) T_{n}(x)}{\sqrt{1-x^{2}}} d x=\frac{\pi}{2} b_{n m},
$$

and then we have

$$
b_{n m}=\frac{2}{\pi} \int_{-1}^{1} \frac{F_{n}(x) T_{m}(x)}{\sqrt{1-x^{2}}} d x .
$$

We define

$$
q_{n k}=\frac{2}{\pi} \int_{0}^{\pi} \cos ^{n} \theta \cos k \theta d \theta .
$$


From reference [6] we know

$$
q_{n k}= \begin{cases}\frac{2 n !}{(n+k) !(n-k) ! !}, & k+n \text { is even and } n \geq k \\ 0, & \text { otherwise }\end{cases}
$$

where $n$ and $k$ are any nonnegative integers. Let $x=\cos \theta$, then we can get the following identity by applying property (2) and property (11):

$$
\begin{aligned}
b_{n m} & =\frac{2}{\pi} \int_{0}^{\pi} \frac{T_{m}(\cos \theta) F_{n}(\cos \theta)}{\sin \theta} \sin \theta d \theta \\
& =\frac{2}{\pi} \sum_{j=1}^{\left\lfloor\frac{n-1}{2}\right\rfloor}\left(\begin{array}{c}
n-j-1 \\
j
\end{array}\right) \int_{0}^{\pi} \cos ^{n-2 j-1} \theta \cos m \theta d \theta \\
& =\sum_{j=1}^{\left\lfloor\frac{n-1}{2}\right\rfloor}\left(\begin{array}{c}
n-j-1 \\
j
\end{array}\right) q_{n-2 j-1, m},
\end{aligned}
$$

and then we have $b_{n m}=0$ if $n+m$ is even. If $n+m$ is odd, we have

$$
\begin{aligned}
b_{n m} & =\sum_{j=1}^{\left\lfloor\frac{n-1}{2}\right\rfloor}\left(\begin{array}{c}
n-j-1 \\
j
\end{array}\right) \cdot \frac{2(n-2 j-1) !}{(n+m-2 j-1) ! !(n-m-2 j-1) ! !} \\
& =\sum_{j=1}^{\left\lfloor\frac{n-1}{2}\right\rfloor} \frac{(n-j-1) !}{j !(n-2 j-1) !} \cdot \frac{2(n-2 j-1) !}{(n+m-2 j-1) ! !(n-m-2 j-1) ! !} \\
& =\sum_{j=1}^{\left\lfloor\frac{n-1}{2}\right\rfloor} \frac{2(n-j-1) !}{j !(n+m-2 j-1) ! !(n-m-2 j-1) ! !} .
\end{aligned}
$$

This proves Lemma 4.

Lemma 5 For any positive integers $m$ and $n$, we have the following identities:

$$
\begin{aligned}
& F_{n}(2 i \cos \theta)=\frac{i^{n+3} \sin n \theta}{\sin \theta}, \\
& \int_{-2 i}^{2 i} \sqrt{x^{2}+4} F_{m}(x) F_{n}(x) d x= \begin{cases}2 i^{2 m-1} \pi, & m=n>0, \\
0, & \text { otherwise. }\end{cases}
\end{aligned}
$$

Proof As we know,

$$
F_{n}(x)=\frac{1}{2^{n} \sqrt{x^{2}+4}}\left[\left(x+\sqrt{x^{2}+4}\right)^{n}-\left(x-\sqrt{x^{2}+4}\right)^{n}\right] .
$$

Let $x=2 i \cos \theta$, then we have

$$
\begin{aligned}
F_{n}(2 i \cos \theta) & =\frac{1}{2 \sin \theta}\left[(i \cos \theta+\sin \theta)^{n}-(i \cos \theta-\sin \theta)^{n}\right] \\
& =\frac{1}{2 \sin \theta}\left(i^{n} e^{-i n \theta}-i^{n} e^{i n \theta}\right)
\end{aligned}
$$




$$
\begin{aligned}
& =\frac{i^{n}}{2 \sin \theta}(\cos n \theta-i \sin n \theta-\cos n \theta-i \sin n \theta) \\
& =\frac{i^{n+3} \sin n \theta}{\sin \theta} .
\end{aligned}
$$

This proves property (12). Let $x=2 i \cos \theta$ in the following identity:

$$
A=\int_{-2 i}^{2 i} \sqrt{x^{2}+4} F_{m}(x) F_{n}(x) d x,
$$

then we can get

$$
\begin{aligned}
A & =\int_{0}^{\pi} 2 \sin \theta F_{n}(2 i \cos \theta) F_{m}(2 i \cos \theta) 2 i \sin \theta d \theta \\
& =\int_{0}^{\pi} 4 i \sin ^{2} \theta \frac{i^{n+3} \sin n \theta}{\sin \theta} \frac{i^{m+3} \sin m \theta}{\sin \theta} d \theta \\
& =4 i^{n+m-1} \int_{0}^{\pi} \sin n \theta \sin m \theta d \theta \\
& =2 i^{n+m-1} \int_{0}^{\pi} \cos (n-m) \theta-\cos (m+n) \theta d \theta .
\end{aligned}
$$

Then we can get property (13). This proves Lemma 5 .

Lemma 6 For any positive integer $n$, we have

$$
\begin{aligned}
& T_{2 n}(x)=\sum_{k=0}^{n} \frac{(-1)^{n-k} \cdot 2^{2 k} \cdot n}{n+k}\left(\begin{array}{c}
n+k \\
2 k
\end{array}\right) x^{2 k}, \\
& T_{2 n+1}(x)=\sum_{k=0}^{n} \frac{(-1)^{n-k} \cdot 2^{2 k} \cdot(2 n+1)}{n+k+1}\left(\begin{array}{c}
n+k+1 \\
2 k+1
\end{array}\right) x^{2 k+1}, \\
& U_{2 n}(x)=\sum_{k=0}^{n} \frac{(-1)^{n-k} \cdot 2^{2 k} \cdot(2 k+1)}{n+k+1}\left(\begin{array}{c}
n+k+1 \\
2 k+1
\end{array}\right) x^{2 k}, \\
& U_{2 n+1}(x)=\sum_{k=0}^{n} \frac{(-1)^{n-k} \cdot 2^{2 k+2} \cdot(k+1)}{n+k+2}\left(\begin{array}{c}
n+k+2 \\
2 k+2
\end{array}\right) x^{2 k+1} .
\end{aligned}
$$

Proof From Theorem 2 of reference [4], we can get the following result easily:

$$
\begin{aligned}
T_{2 n}(x) & =\sum_{k=0}^{n}(-1)^{n-k}\left[2^{2 k}\left(\begin{array}{c}
n+k \\
n-k
\end{array}\right)-2^{2 k-1}\left(\begin{array}{c}
n+k-1 \\
2 k-1
\end{array}\right)\right] x^{2 k} \\
& =\sum_{k=0}^{n}(-1)^{n-k} \cdot 2^{2 k-1}\left[\frac{2(n+k) !}{(2 k) !(n-k) !}-\frac{(n+k-1) !}{(2 k-1) !(n-k) !}\right] x^{2 k} \\
& =\sum_{k=0}^{n}(-1)^{n-k} \cdot 2^{2 k} \frac{n(n+k-1) !}{(2 k) !(n-k) !} x^{2 k} \\
& =\sum_{k=0}^{n} \frac{(-1)^{n-k} \cdot 2^{2 k} \cdot n}{n+k}\left(\begin{array}{c}
n+k \\
2 k
\end{array}\right) x^{2 k} .
\end{aligned}
$$


From Theorem 2 of reference [4], we know

$$
T_{2 n+1}(x)=\sum_{k=0}^{n}(-1)^{n-k}\left[2^{2 k+1}\left(\begin{array}{c}
n+k+1 \\
n-k
\end{array}\right)-2^{2 k}\left(\begin{array}{c}
n+k \\
2 k
\end{array}\right)\right] x^{2 k+1}
$$

In a similar way, we can get

$$
T_{2 n+1}(x)=\sum_{k=0}^{n} \frac{(-1)^{n-k} \cdot 2^{2 k} \cdot(2 n+1)}{n+k+1}\left(\begin{array}{c}
n+k+1 \\
2 k+1
\end{array}\right) x^{2 k+1}
$$

We can get $T_{n}^{\prime}(x)=n U_{n-1}(x)$ easily from the definition of the Chebyshev polynomials. If we derive both sides of the above properties, we will get

$$
\begin{aligned}
& U_{2 n}(x)=\sum_{k=0}^{n} \frac{(-1)^{n-k} \cdot 2^{2 k} \cdot(2 k+1)}{n+k+1}\left(\begin{array}{c}
n+k+1 \\
2 k+1
\end{array}\right) x^{2 k}, \\
& U_{2 n+1}(x)=\sum_{k=0}^{n} \frac{(-1)^{n-k} \cdot 2^{2 k+2} \cdot(k+1)}{n+k+2}\left(\begin{array}{c}
n+k+2 \\
2 k+2
\end{array}\right) x^{2 k+1} .
\end{aligned}
$$

This proves Lemma 6.

Lemma 7 For any positive integer $n$, let

$$
T_{2 n}(x)=\sum_{s=1}^{+\infty} c_{2 n, s} F_{s}(x)
$$

and

$$
T_{2 n-1}(x)=\sum_{s=1}^{+\infty} c_{2 n-1, s} F_{s}(x)
$$

then we can get

$$
\begin{aligned}
& c_{2 n, s}= \begin{cases}\sum_{k=0}^{n} \frac{2^{4 k+1} s n \cdot i^{3 s+2 n+1}(n+k-1) !}{(n-k) !(2 k+s+1) !(2 k-s+1) ! !}, & \text { s is odd, } \\
0, & \text { otherwise, }\end{cases} \\
& c_{2 n-1, s}= \begin{cases}\sum_{k=1}^{n} \frac{(2 n s-s) i^{3 s+2 n}(n+k-2) !}{2^{2-4 k}(n-k) !(2 k+s) !(2 k-s) ! !}, & \text { s is even, } \\
0, & \text { otherwise. }\end{cases}
\end{aligned}
$$

Proof At first, we multiply $\sqrt{x^{2}+4} F_{m}(x)$ to both sides of (14), then integrate it from $-2 i$ to $2 i$. We can get the following identity by applying Lemma 5 , where $m$ is any positive integer:

$$
\int_{-2 i}^{2 i} \sqrt{x^{2}+4} F_{m}(x) T_{2 n}(x) d x=\sum_{s=1}^{\infty} \int_{-2 i}^{2 i} c_{2 n, s} \sqrt{x^{2}+4} F_{s}(x) F_{m}(x) d x=2 i^{2 m-1} \pi c_{2 n, m}
$$

and then we have

$$
c_{2 n, m}=\frac{(-i)^{2 m-1}}{2 \pi} \int_{-2 i}^{2 i} \sqrt{x^{2}+4} F_{m}(x) T_{2 n}(x) d x .
$$


Let $x=2 i \cos \theta$, then we can get the following identity by applying Lemma 5 and property (10):

$$
\begin{aligned}
c_{2 n, m} & =\frac{(-i)^{2 m-1}}{2 \pi} \int_{0}^{\pi} T_{2 n}(2 i \cos \theta) F_{m}(2 i \cos \theta) 4 i \sin ^{2} \theta d \theta \\
& =\frac{-i^{2 m}}{2 \pi} \int_{0}^{\pi} T_{2 n}(2 i \cos \theta) \cdot \frac{i^{m+3} \sin m \theta}{\sin \theta} \cdot(2 \sin \theta)^{2} d \theta \\
& =\frac{2 i^{3 m+1}}{\pi} \int_{0}^{\pi} T_{2 n}(2 i \cos \theta) \sin m \theta \sin \theta d \theta \\
& =\sum_{k=0}^{n} \frac{2^{4 k} \cdot n \cdot i^{3 m+2 n+1}}{n+k}\left(\begin{array}{c}
n+k \\
2 k
\end{array}\right) w_{2 k, m-1},
\end{aligned}
$$

so when $m$ is even, we have $c_{2 n, m}=0$. When $m$ is odd, we have

$$
\begin{aligned}
c_{2 n, m} & =\sum_{k=0}^{n} \frac{2^{4 k} \cdot n \cdot i^{3 m+2 n+1}}{n+k} \cdot\left(\begin{array}{c}
n+k \\
2 k
\end{array}\right) \cdot \frac{2 m \cdot(2 k) !}{(2 k+m+1) ! !(2 k-m+1) ! !} \\
& =\sum_{k=0}^{n} \frac{2^{4 k} \cdot n \cdot i^{3 m+2 n+1}}{n+k} \cdot \frac{(n+k) !}{(2 k) !(n-k) !} \cdot \frac{2 m \cdot(2 k) !}{(2 k+m+1) ! !(2 k-m+1) ! !} \\
& =\sum_{k=0}^{n} \frac{2^{4 k+1} \cdot m n \cdot i^{3 m+2 n+1}(n+k-1) !}{(n-k) !(2 k+m+1) ! !(2 k-m+1) ! !}
\end{aligned}
$$

In a similar way, we can get the following result easily:

$$
\begin{aligned}
c_{2 n-1, m} & =\frac{(-i)^{2 m-1}}{2 \pi} \int_{0}^{\pi} T_{2 n-1}(2 i \cos \theta) F_{m}(2 i \cos \theta) 4 i \sin ^{2} \theta d \theta \\
& =\sum_{k=1}^{n} \frac{2^{4 k-3} \cdot(2 n-1) \cdot i^{3 m+2 n}}{n+k-1}\left(\begin{array}{c}
n+k-1 \\
2 k-1
\end{array}\right) w_{2 k-1, m-1},
\end{aligned}
$$

and we have $c_{2 n-1, m}=0$ if $m$ is odd. If $m$ is even, we have

$$
\begin{aligned}
c_{2 n-1, m} & =\sum_{k=1}^{n} \frac{2^{4 k-3} \cdot(2 n-1) \cdot i^{3 m+2 n}}{n+k-1}\left(\begin{array}{c}
n+k-1 \\
2 k-1
\end{array}\right) \frac{2 m(2 k-1) !}{(2 k+m) ! !(2 k-m) ! !} \\
& =\sum_{k=1}^{n} \frac{(2 n m-m) i^{3 m+2 n}(n+k-2) !}{2^{2-4 k}(n-k) !(2 k+m) ! !(2 k-m) ! !} .
\end{aligned}
$$

This proves Lemma 7.

Lemma 8 For any positive integer $n$, let

$$
U_{2 n}(x)=\sum_{s=1}^{+\infty} d_{2 n, s} F_{s}(x)
$$

and

$$
U_{2 n-1}(x)=\sum_{s=1}^{+\infty} d_{2 n-1, s} F_{s}(x),
$$


then we can get

$$
\begin{aligned}
& d_{2 n, s}= \begin{cases}\sum_{k=0}^{n} \frac{2^{4 k+1} \cdot i^{3 s+2 n+1} s(n+k) !}{(n-k) !(2 k+s+1) !(2 k-s+1) ! !}, & \text { s is odd, } \\
0, & \text { otherwise, }\end{cases} \\
& d_{2 n-1, s}= \begin{cases}\sum_{k=0}^{n-1} \frac{2^{4 k+3} \cdot i^{3 s+2 n} s(n+k-1) !}{(n-k-1) !(2 k+s+2) !(2 k+2-s) ! !}, & \text { s is even, } \\
0, & \text { otherwise. }\end{cases}
\end{aligned}
$$

Proof At first, we multiply $\sqrt{x^{2}+4} F_{m}(x)$ to both sides of (18), then integrate it from $-2 i$ to $2 i$, we can get the following identities by applying Lemma 5 , where $m$ is any positive integer:

$$
\int_{-2 i}^{2 i} \sqrt{x^{2}+4} F_{m}(x) U_{2 n}(x) d x=\sum_{k=1}^{\infty} \int_{-2 i}^{2 i} d_{n s} \sqrt{x^{2}+4} F_{s}(x) F_{m}(x) d x=2 i^{2 m-1} \pi d_{2 n, m}
$$

then we have

$$
d_{2 n, m}=\frac{(-i)^{2 m-1}}{2 \pi} \int_{-2 i}^{2 i} \sqrt{x^{2}+4} F_{m}(x) U_{2 n}(x) d x .
$$

Let $x=2 i \cos \theta$, then we can get the following identity by applying Lemma 5 and property (10):

$$
\begin{aligned}
d_{2 n, m} & =\frac{i^{2 m+1}}{2 \pi} \int_{0}^{\pi} U_{2 n}(2 i \cos \theta) F_{m}(2 i \cos \theta) 4 i \sin ^{2} \theta d \theta \\
& =\frac{i^{2 m+2}}{2 \pi} \int_{0}^{\pi} U_{2 n}(2 i \cos \theta) \frac{i^{m+3} \sin m \theta}{\sin \theta}(2 \sin \theta)^{2} d \theta \\
& =\frac{2 i^{3 m+1}}{\pi} \int_{0}^{\pi} U_{2 n}(2 i \cos \theta) \sin m \theta \sin \theta d \theta \\
& =\sum_{k=0}^{n} \frac{2^{4 k} \cdot(2 k+1) \cdot i^{3 m+1+2 n}}{n+k+1}\left(\begin{array}{c}
n+k+1 \\
2 k+1
\end{array}\right) w_{2 k, m-1},
\end{aligned}
$$

so we have $d_{2 n, m}=0$ if $m$ is even. If $m$ is odd, we have

$$
\begin{aligned}
d_{2 n, m} & =\sum_{k=0}^{n} \frac{2^{4 k} \cdot(2 k+1) \cdot i^{3 m+1+2 n}}{n+k+1}\left(\begin{array}{c}
n+k+1 \\
2 k+1
\end{array}\right) \cdot \frac{2 m \cdot(2 k) !}{(2 k+m+1) ! !(2 k-m+1) ! !} \\
& =\sum_{k=0}^{n} \frac{2^{4 k} \cdot(2 k+1) \cdot i^{3 m+1+2 n}}{n+k+1} \cdot \frac{(n+k+1) !}{(2 k+1) !(n-k) !} \cdot \frac{2 m \cdot(2 k) !}{(2 k+m+1) ! !(2 k-m+1) ! !} \\
& =\sum_{k=0}^{n} \frac{2^{4 k+1} \cdot i^{3 m+2 n+1} m(n+k) !}{(n-k) !(2 k+m+1) ! !(2 k-m+1) ! !}
\end{aligned}
$$

In a similar way, we have

$$
\begin{aligned}
d_{2 n-1, m} & =\frac{(-i)^{2 m-1}}{2 \pi} \int_{0}^{\pi} U_{2 n-1}(2 i \cos \theta) F_{m}(2 i \cos \theta) 4 i \sin ^{2} \theta d \theta \\
& =\sum_{k=0}^{n-1} \frac{2^{4 k+3} \cdot(k+1) \cdot i^{3 m+2 n}}{n+k+1}\left(\begin{array}{c}
n+k+1 \\
2 k+2
\end{array}\right) w_{2 k+1, m-1}
\end{aligned}
$$


When $m$ is odd, we have $d_{2 n-1, m}=0$. When $m$ is even, we have

$$
\begin{aligned}
d_{2 n-1, m} & =\sum_{k=0}^{n-1} \frac{2^{4 k+3} \cdot(k+1) \cdot i^{3 m+2 n}}{n+k+1}\left(\begin{array}{c}
n+k+1 \\
2 k+2
\end{array}\right) \frac{2 m(2 k+1) !}{(2 k+m+2) ! !(2 k+2-m) ! !} \\
& =\sum_{k=0}^{n-1} \frac{2^{4 k+3} \cdot i^{3 m+2 n} m(n+k-1) !}{(n-k-1) !(2 k+m+2) ! !(2 k+2-m) ! !} .
\end{aligned}
$$

This proves Lemma 8.

\section{Proof of the theorems and corollaries}

In this section, we will prove our theorems and corollaries. First of all, we can prove all the theorems from Lemma 4, Lemma 7 and Lemma 8 easily.

Proof of Corollary 1 We can get the following properties from Lemma 2 and Lemma 3 by letting $x=T_{m}(x)$ in Theorem 3:

$$
\begin{aligned}
& F_{2 n-1}\left(T_{m}(x)\right)=\sum_{k=0}^{n-1} \sum_{j=1}^{n-1} \frac{2^{2 j+2-2 n}(2 k+1)(n-j-1) !}{j !(n-k-j-1) !(n+k-j) !} U_{2 k}\left(T_{m}(x)\right), \\
& F_{2 n}\left(T_{m}(x)\right)=\sum_{k=1}^{n-1} \sum_{j=1}^{n-1} \frac{2^{2 j-2 n+2} k(2 n-j-1) !}{j !(n-k-j) !(n+k-j) !} U_{2 k}\left(T_{m}(x)\right) .
\end{aligned}
$$

Then, taking $x=\frac{i}{2}$ in the above identities, according to Lemma 2, we can get Corollary 1 .

Proof of Corollary 2 We can get the following properties from Lemma 2 and Lemma 3 by letting $x=T_{m}(x)$ in Theorem 4:

$$
\begin{aligned}
F_{2 n}\left(T_{m}(x)\right)= & \sum_{k=1}^{n} \sum_{j=1}^{n-1} \frac{2^{2 j+2-2 n}(2 n-j-1) ! T_{2 k-1}\left(T_{m}(x)\right)}{j !(2 n+2 k-2 j-2) !(2 n-2 k-2 j) !}, \\
F_{2 n-1}\left(T_{m}(x)\right)= & \sum_{k=1}^{n-1} \sum_{j=1}^{n-1} \frac{2^{2 j+3-2 n}(2 n-j-2) ! T_{2 k}\left(T_{m}(x)\right)}{j !(n+k-j-1) !(n-k-j-1) !} \\
& +\sum_{j=1}^{n-1} \frac{2^{2 j+2-2 n}(2 n-j-2) !}{j !(n-j-1) !(n-j-1) !} T_{0}\left(T_{m}(x)\right) .
\end{aligned}
$$

Then, taking $x=\frac{i}{2}$ in the above identities, according to Lemma 2, we can get Corollary 2 .

The author declares that there is no conflict of interests regarding the publication of the article.

\section{Acknowledgements}

The author would like to thank the referees for their very helpful and detailed comments which have significantly

improved the presentation of this paper.

Received: 28 November 2014 Accepted: 18 February 2015 Published online: 09 April 2015 
References

1. Zhang, W: Some identities involving the Fibonacci numbers and Lucas numbers. Fibonacci Q. 42, 149-154 (2004)

2. Falcón, S, Plaza, Á: On k-Fibonacci sequences and polynomials and their derivatives. Chaos Solitons Fractals 39 , 1005-1019 (2009)

3. Falcón, S, Plaza, Á: The k-Fibonacci sequences and the Pascal 2-triangle. Chaos Solitons Fractals 38, 38-49 (2007)

4. Wu, X, Yang, G: The general formula of Chebyshev polynomials. J. Wuhan Transp. Univ. 24, 573-576 (2000)

5. Abramowitz, M, Stegun, IA: Handbook of Mathematical Functions: With Formulas, Graphs and Mathematical Tables, chap. 22, pp. 771-802. Dover New York (1965)

6. Ma, R, Zhang, W: Several identities involving the Fibonacci numbers and Lucas numbers. Fibonacci Q. 5, 164-171 (2007)

Submit your manuscript to a SpringerOpen ${ }^{\bullet}$ journal and benefit from:

- Convenient online submission

Rigorous peer review

- Immediate publication on acceptance

- Open access: articles freely available online

- High visibility within the field

- Retaining the copyright to your article 